\title{
Histopathological study of neoplastic lesions of large intestine -A five year experience
}

\author{
Aneeta Singh Malhotra ${ }^{1, *}$, Archana Gupta ${ }^{2}$, Sheikh Mahvesh $^{3}$, Anam Khurshied ${ }^{4}$, Urvashi Andotra ${ }^{5}$ \\ ${ }^{1,2}$ Associate Professor, ${ }^{3,4} 3^{\text {rd }}$ Year Post Graduate, ${ }^{5} 2^{\text {nd }}$ Post Graduate, Dept. of Pathology, Acharya Shri Chander College of \\ Medical Sciences \& Hospital, Sidhra, Jammu, India
}

*Corresponding Author:

Email: aneetasinghmalhotra@gmail.com

Received: $1^{\text {st }}$ November, 2017

Accepted: $17^{\text {th }}$ March, 2018

\begin{abstract}
Introduction: Large intestine has a varied spectrum of lesions both non-neoplastic and neoplastic. We took both a retrospective and prospective study in the Post Graduate Department of Pathology. The clinical details were sought out from the medical records and the specimens were collected from the patients diagnosed as colorectal carcinoma in the histopathology spectrum. Intestinal tumors account for a large proportion of all neoplasms. There is a significant geographical, racial and ethnic variation in the incidence, rate and pattern of colorectal carcinoma, it is third most common cancer in men and second in women worldwide.

Aims and Objectives: (1). To study the neoplastic lesions of large intestine for a period of five years; (2). To evaluate different histopathological types of tumors of colorectum; (3). To correlate the various neoplastic lesions in relation to age, sex, family history and dietary habits of the patient.

Materials and Methods: A five year study from year December 2012 to December 2017, 3 year retrospective and 2 years prospective study was carried out. The necessary information and clinical data was obtained from the concerned sections. It included 248 resected specimens of large intestine.

Results: Out of 248 large intestine specimens, $30.65 \%$ i.e 76 were non-neoplastic, $62.1 \%$ i.e 154 were neoplastic and 18 were inadequate biopsies.

Malignant Neoplasm is more common than benign neoplasm in the colon. Adenocarcinoma is the most common histological variant of colon carcinoma. There is marked increase in the incidence of malignancies of colon, hence the approach to early diagnosis by carrying out biopsies in clinically symptomatically suspicious patients should be the thumb rule for good management.
\end{abstract}

Keywords: Neoplasm, Adenoma, Adenocarcinoma, Polyp, Gastrointestinal stromal tumor.

\section{Introduction}

Colorectal carcinoma is the third leading cancer worldwide, approximately accounting for $9 \%$ of all cancers. The estimated worldwide annual incidence of colorectal carcinoma is 1 million, which makes it the second most common carcinoma in females and third in males. ${ }^{1}$ Mean age of diagnosis is 62 years with a slight higher incidence among the males as compared to the females. Generally, the incidence of colorectal carcinoma and the mortality rates are greater in developed nations of Western world, ${ }^{2}$ that is north America, Australia and Europe whereas the developing continents like Africa and Africa have lower rate of occurance. Lack of physical activity, red meat diet, high fat diet with poor fiber may be related to the increase in incidence. The varied dietary and environmental exposures explain the geographic differences for the occurance of colorectal cancers. High predisposition for colorectal carcinoma in patients with familial adenomatous polyposis (nearly $100 \%$ by 50 years of age) is because of genetic factors. About $60 \%$ of colorectal cancer occurs in developing countries. Five year survival rate in colorectal cancer is about $60-95 \%$ in the initial stages and decreases dramatically to $30 \%$ in stages where lymph node metastasis are detected. ${ }^{5}$
The most common malignancy arising from colorectal region is adenocarcinoma. Benign lesions are nonneoplastic polyps that is hyperplastic, hamartomatous, juvenile, peutz jeghar, inflammatory and lymphoid. Adenoma, neuroma, angioma, lipoma are other benign lesions, ulcerative colitis which is a inflammatory bowel disease and is premalignant lesion.

\section{Materials and Methods}

A three year retrospective study was done from December 2012 to November 2015 and two year prospective study was conducted with effect from December 2015 to November 2017. Hence it is a five year study altogether. From the record section of pathology department, proper ethical clearance and relevant clinical information was taken. The corresponding slides and tissue blocks were retrieved and microscopic analysis was carried out. The specimen and biopsies of neoplastic lesions of large intestine for prospective study were fixed in $10 \%$ buffered formalin and then detail gross examination was done, histopathological processing and section blocks were made and stained with hematoxylin and eosin and mounted indistyreneplasticiser xylene (DPX). The final diagnosis was given after the detailed study of sections 
under the light microscope. Then, data was analysed and results were obtained.

\section{Results}

In our study, there were 248, which included biopsis and resected specimens. Out of 248 cases of large intestine, 76 cases $(30.65 \%)$ were benign, 154
$(62.1 \%)$ were malignant while $18(7.26 \%)$ cases were inadequate. The non-neoplastic lesions included the infective, ischemic lesions and various congenital anomalies. Age from 15-88 years with mean age of 56 years was taken. Most of the patients of the colorectal carcinoma were non-vegetarians. $20(30.8 \%)$ of cases had a family history of colorectal carcinoma.

Table 1: Age incidence of all 154 cases of neoplastic lesions in present study

\begin{tabular}{|l|c|c|c|}
\hline Age in years & Number of cases & Male & Female \\
\hline $0-10$ & 3 & 2 & 1 \\
\hline $11-20$ & 7 & 4 & 3 \\
\hline $21-30$ & 6 & 3 & 3 \\
\hline $31-40$ & 8 & 4 & 4 \\
\hline $41-50$ & 21 & 11 & 10 \\
\hline $51-60$ & 42 & 22 & 20 \\
\hline $61-70$ & 45 & 27 & 18 \\
\hline $71-80$ & 20 & 10 & 10 \\
\hline $81-90$ & 2 & 2 & 0 \\
\hline total & 154 & $85(55.194 \%)$ & $69(44.805 \%)$ \\
\hline
\end{tabular}

In the present study, rectum was the most common location involved with $40(25.8 \%)$ cases of the total 154 carcinoma of colorectum, followed closely by sigmoid colon with $34(22.5 \%)$ cases followed by rest of the segments of the colon.

Regarding gender distribution, in our study; there was a higher incidence in the number of males affected by colorectal malignant $(85,55.194 \%)$ than females $(69,44.805 \%)$. Analysis of incidence per decade of age showed highest figure between 61-70 years. Two cases were of gastrointestinal stromal tumor (GIST). Immunohistochemistry with CD117 for GIST was positive for GIST. A single case of non-Hodgkins lymphoma of diffuse large B-cell Lymphoma was found, it was confirmed with immunohistochemistry CD19 and CD20.

The most common morphology of tumor was adenocarcinoma 151 cases $(98.05 \%)$, out of total 151 adenocarcinoma a number of $45(29.801 \%)$ were well differentiated adenocarcinoma, $85(56.291 \%)$ were moderately differentiated and $21(13.907 \%)$ were poorly differentiated adenocarcinoma. Well differentiated tumors look more like the glandular tissue that they are derived from, they tend to grow and spread more slowly. As compared to well differentiated and moderately differentiated colon carcinoma, poorly differentiated colon tumors are more aggressive and tend to spread quickly. The histological grading of colon cancer is important in determining in patients prognosis as to how far the tumor has spread. As we know $90 \%$ of colorectal carcinoma are adenocarcinomas. Epithelial cells of colorectal mucosa give rise to adenocarcinomas, which is the most common form. Other variants include neuroendocrine squamous cell, adenosquamous, spindle cell and undifferentiated carcinomas. The common type of adenocarcinoma presents with gland formation. It's the gland formation which forms the basis for histological grading of the tumor. In well differentiated adenocarcinoma there is more than $95 \%$ of gland formation, in moderately differentiated adenocarcinoma gland formation is seen upto $50-95 \%$ while in poorly differentiated adenocarcinoma gland formation is less than 50\%. The grading is an important prognostic marker, as poor patient survival is seen with high grade tumor. The most important work of pathologist is to look for evidence of invasion. The invasive carcinoma disrupts and invades through the muscularis mucosae into the submucosa. There is also an associated desmoplastic reaction of the stroma, with accompanying necrosis. Invasive carcinoma always denotes an invasion into the submucosa of the colorectum.

Mucinous Adenocarcinoma: This variant of adenocarcinoma shows a fungating mass on gross examination, while on microscopy show round to polygonal tumor cells with hyperchromatic nuclei, showing glandular pattern. The tumor cells are seen floating in extensive pools of mucin. There has to be more than $50 \%$ of tumor volume which is composed of extracellular mucin. Also a variable number of signet ring cells are seen.

Gastro-intestinal Stromal Tumors (GIST): GISTs are not a common tumor of the gastrointestinal tract. Intestinal cells of cajal or pacemaker cells of gastrointestinal muscularis propria give rise to these tumors. Their presentation in the colon is very rare. Grossly, GIST presents as a well circumscribed, fleshy, big intramural tumor. Hemorrhage and necrosis may be seen. These are either spindle cell GISTs or Epithelioid GISTs. Spindle cell GIST present as intersecting fascicles of spindle cells with eosinophilic cytoplasm 
against an edematous stroma. Epithelioid GISTs presents as rounded epithelioid cells with muscle infiltration. The most useful diagnostic marker is KIT which is immunohistochemically seen in $95 \%$ of these tumors.

Diffuse, Large B cell Lymphoma: Colorectal lymphomas are rare and can either presents as primary or secondary to a systemic disease. Clinical presentation is usually pain and obstruction of abdomen. Among all colorectal lymphomas, diffuse B cell is the most common histological subtype. Most patients undergo surgery but multiagent chemotherapy remains the mainstay of treatment.
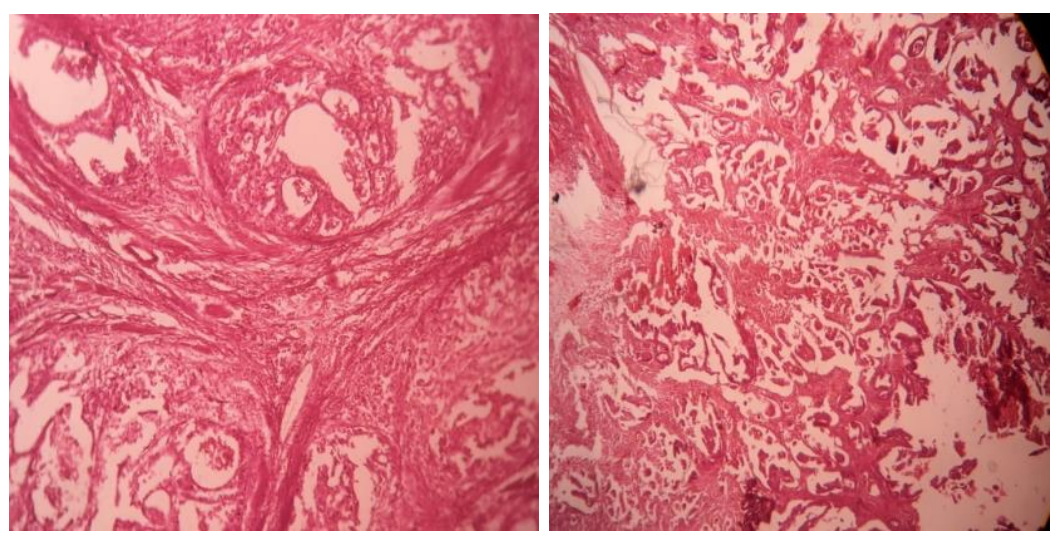

Fig. 1: Photograph showing histopathological features of moderately differentiating adenocarcinoma colon
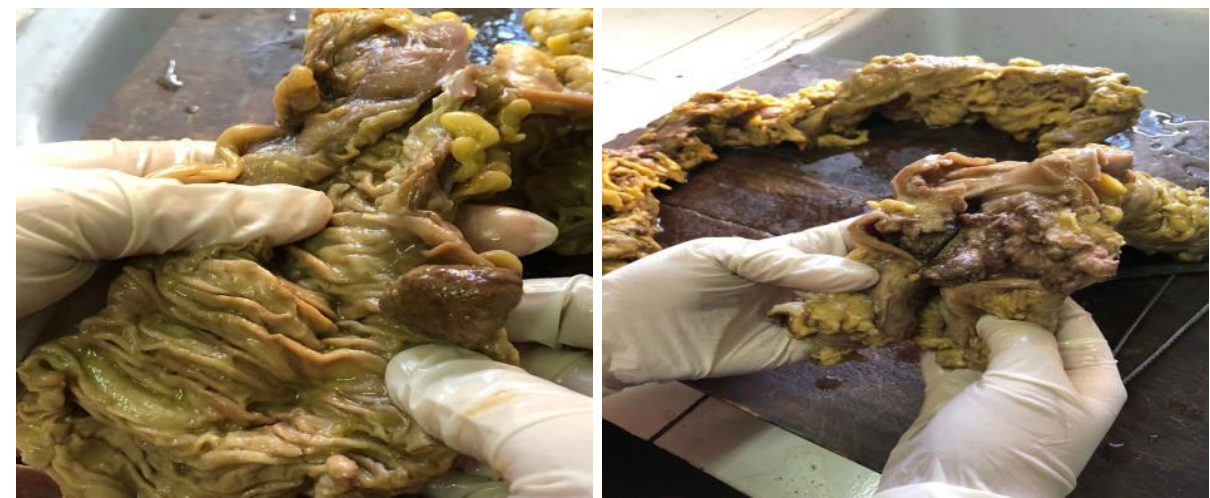

Fig. 2: Photograph showing gross image of a) sessile polyp b) fungating growth colon

\section{Discussion}

The peak age group of colorectal carcinoma is in $6^{\text {th }}$ and $7^{\text {th }}$ decade $^{6}$ with moderately differentiated adenocarcinoma as the most common type. ${ }^{7}$ In our study also reported moderately differentiated adenocarcinoma being the commonest type $(51.83 \%)$. In our study there was slight male predominance with male to female ratio of 1.3:1 which was found in other studies as well. ${ }^{8}$ Inspite of all the advanced techniques, the incidence of colorectal carcinoma, in increasing ${ }^{9}$ with highest incidence in Australia, New Zealand, Europe and North America, the lowest incidence being reported in Africa and Asia. ${ }^{10}$ A large number of patients presented with bleeding PR as the presenting symptom. Other associated symptoms included abdominal pain, anemia, altered bowel habits and intestinal obstruction.

In our study, most of the patients had somewhat inactive way of life (sedentary job) and more intake of red meat and low fiber diet was observed. Also reduced physical activity, smoking and increased alcohol consumption leads to rise in number of cases of colorectal carcinoma. ${ }^{12}$ The carcinoma of left side colon exceeds the carcinoma of right side which again is consistent with world's literature and other studies. ${ }^{13}$ The moderately differentiated tumors were more.

\section{Conclusion}

Colorectal Carcinoma was diagnosed especially in people of middle age and seen more in males, 154 patients were studied which showed increase frequency of rectum carcinoma as compaired to other segments of colon. The histopathological analysis/study depicted about $98 \%$ of colorectal malignancies as adenocarcinomas, mostly presenting as moderate differentiation. Most cancers were recorded in the rectum and left colon. There was an increased no. of cases among females. As the incidence of coloncarcinoma is increasing due to altered lifestyle, people with altered bowel symptoms, iron deficiency anemia should be evaluated. 


\section{References}

1. Rasool MU, Mubeen B, Andrabi RS, Hamis S, Rasool Z, Shah P, Shah OJ,2015 : Histopathological study of neoplastic lesions of large intestine, Int. Res. J. Medical Sci, may 2015, 3(5):1-5.

2. Parkin DM, Pisani P and Ferlay J., Global cancer statistics, CA cancer J Clin. 1999, 49(1), 33.

3. Siegel R, Ward, Brawley O, Jemal A, Cancer Statistics, 2011: the impact of eliminating socio-economic and racial disparities on premature cancer deaths, cancer $J$ clin, 2011, 61(4):212-236.

4. Fleming M, Ravnla S, Tatishchev SF, Wang HL, Colorectal Carcinoma : Pathologic aspects, J Gastrointest Oncol, 2012, 3(3):153-173.

5. Kanthan R, Senger JL, Kanthan Sc, Molecular events in primary and Metastatic colorectal carcinoma: A review, Pathology Res Int, 2012:597497.

6. Priyank Sharma, Muktanjalee Deka. A study of neoplastic lesions of Colorectum in a Tertiary Care Hospital, International Journal of Scientific Study. Nov 2015(3)8, pp.88-91.

7. Caliskan C, Guler N, karaca C, Makay O, Firat O, Korkut MA. Negative prognostic factors in colorectal carcinoma: an analysis of 448 patients. Indian J. Surg, 2010;72:24348.

8. Gul J, Zargar SA and Rather S et al, Incidence of colorectal cancer in Kashmir Valley, India. Indian Journal Gastro entrology, 30 (10):7-11.
9. Troeung L, Berry NS, Martin A, Malacova E, Ee H, Leary PO, Lansdrop-Vogelaar I, Preen DB, Increasing incidence of colorectal carcinoma in adoloscents and young adults Aged 15-39 years in western Australia 1982-2007. Fpubh, 2017;5:1-8.

10. Jemal A, Bray F, Center MM, Ferlay J, Ward E, Forman d, Global Cancer Statistics, CA Cancer J clin, 2011, 61 (2):69-90.

11. Slattery ML. Potter J and Caan S et al, Energy balance and colon cancer beyond physical activity, cancer Res, 57(1), 75(1997).

12. Durko L, Malecha - Panas E, Lifestyle modifications and colorectal cancer Rep, 2014,10:45-54.

13. Xu A.G, Yu Z.J. and Jiang B et al, colorectal cancer in Guangdong Province of China: A demographic and anatomic survey, world Journal Gastro enterology, 16(8), 960-965(2010).

How to cite this article: Malhotra AS, Gupta A, Mahvesh S, Khurshied A, Andotra U. Histopathological study of neoplastic lesions of large intestine $-\mathrm{A}$ five year experience. Ind $\mathrm{J}$ Pathol Oncol, 2018;5(3):454-457. 